\title{
A photometric study of the intermediate age open cluster King $5^{\star}$
}

\author{
G. Carraro ${ }^{1}$ and A. Vallenari ${ }^{2}$ \\ 1 Dipartimento di Astronomia, Universitá di Padova, vicolo dell'Osservatorio 5, I-35122 Padova, Italy \\ 2 Osservatorio Astronomico di Padova, vicolo Osservatorio 5, I-35122 Padova, Italy \\ e-mail: carraro,vallenari@pd.astro.it
}

Received June 30; accepted November 19, 1999

\begin{abstract}
We report on near IR ( $J$ and $K$ bands) observations of an $8^{\prime} \times 8^{\prime}$ region centered on the poorly studied open cluster King 5, for which only optical photometry existed. Photometry of two nearby fields is also reported. We found that the cluster is of moderate age (1.0 Gyr old), intermediate in age between the Hyades and NGC 752. Combining optical and IR photometry we obtain estimates for the cluster parameters. The color excesses $E(J-K), E(V-I)$ and $E(V-K)$ are $0.50,1.10$ and 2.45 , respectively. The true distance modulus turns out to be $(m-M)_{0}=11.40 \pm 0.15$. As a consequence, King 5 is $1.9 \mathrm{kpc}$ far from the Sun.
\end{abstract}

Key words: infrared: stars - techniques: photometry open clusters and associations: King 5 - open clusters and associations: general

\section{Introduction}

This paper continues a series dedicated to the presentation of near-infrared photometry (in $J$ and $K$ pass-bands) for northern galactic open clusters. We already reported on the very young open clusters NGC 1893 and Berkeley 86 (Vallenari et al. 1999a), the old clusters Berkeley 17 and Berkeley 18 (Carraro et al. 1999a), and the intermediate age clusters IC 166 and NGC 7789 (Vallenari et al. 1999b). Here we present $J$ and $K$ photometry for King 5 , a poorly studied open cluster for which no detailed study has been reported so far.

King 5 is located near the galactic plane at $l=143.75$ and $b=-4.27$, and it is designated also as OCL 384 and $\mathrm{C} 0311+525$ by IAU. Its diameter is estimated to be about $5^{\prime}-8^{\prime}$.

Send offprint requests to: G. Carraro, e-mail: carraro@pd.astro.it

* Based on observations taken at TIRGO.
A preliminary investigation was conducted by Phelps et al. (1994) who obtained VI photometry for 1343 stars in a region of $11^{\prime} \times 11^{\prime}$. This study revealed that King 5 is a cluster of the Hyades generation, with an age around one billion years, derived from a morphological age estimator.

These authors do not report estimates for cluster distance and reddening.

Moderate resolution spectra for 24 stars in the field of King 5 have been obtained by Scott et al. (1995). From this analysis it emerges that King 5 belongs to the old thin disk population, and its rotational velocity around the galactic center $\left(-52 \mathrm{~km} \mathrm{~s}^{-1}\right)$ is consistent with Hron (1987) rotation curve at the cluster location.

On the base of the radial velocities 19 members have been singled out.

More recently Durgapal et al. (1998) published $U B V R I$ photometry of King 5, inferring by comparison with solar metallicity isochrones an age slightly lower than 1 Gyr, a distance of $2 \mathrm{kpc}$ and a reddening $E(V-I)=0.94$.

Finally it is worth recalling that the metal abundance of King 5 has been determined by Friel et al. (1995) using medium resolution spectroscopy of twelve giant stars. From this study $[\mathrm{Fe} / \mathrm{H}]$ turns out to be $(-0.38 \pm 0.20)$, half the solar value.

In this paper we combine together IR and optical photometry to obtain an estimate of the cluster fundamental parameters. We find that the cluster is 1.0 Gyr old.

The plan of the paper is as follows: Sect. 2 is devoted to the presentation of data acquisition and reduction; in Sect. 3 we discuss the morphology of the Color Magnitude Diagrams (CMDs) for different pass-bands; Sect. 4 is dedicated to the derivation of the color excess, while age and distance are inferred in Sect. 5. Finally our conclusions are summarized in Sect. 6. 
Table 1. Observation log book

\begin{tabular}{c|c|c|c|c|c}
\hline \hline Cluster & $\alpha$ & $\delta$ & Date & \multicolumn{2}{|c}{ Exposure Times (sec) } \\
& $(2000)$ & $(2000)$ & & $J$ & $K$ \\
\hline King 5 & 31447 & 524239 & Oct. 25, 1997 & 540 & 880 \\
King 5 F1 & 31423 & 524839 & Oct. 27, 1997 & 480 & 620 \\
King 5 F2 & 31542 & 523317 & Oct. 27, 1997 & 480 & 620 \\
K5A & 31440 & 524251 & Oct. 25, 1997 & & \\
K5B & 31427 & 524202 & Oct. 25, 1997 & & \\
\hline \hline
\end{tabular}

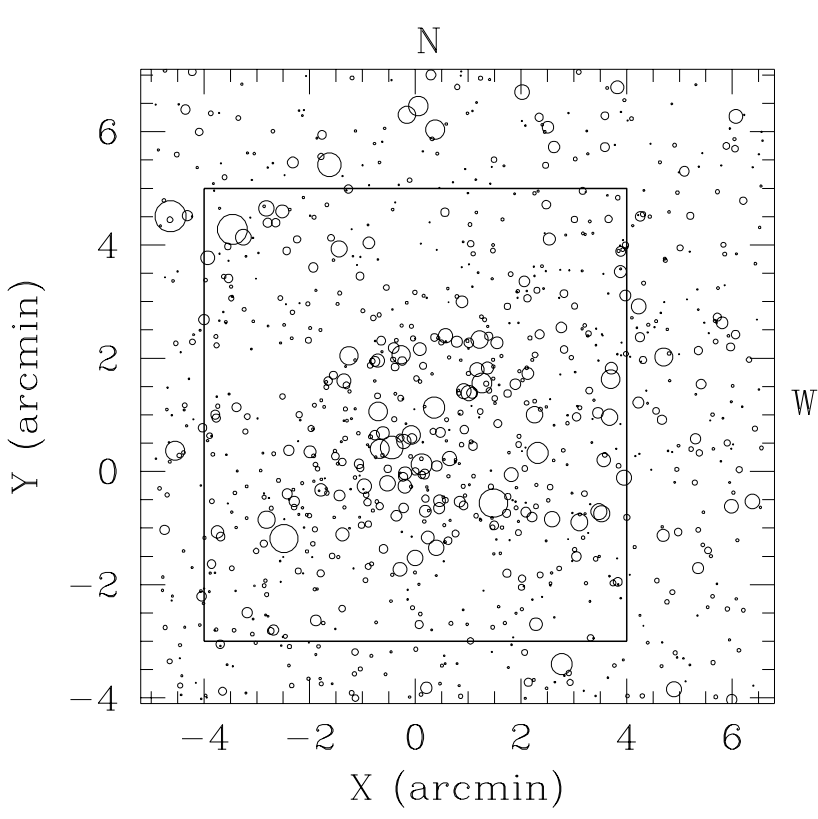

Fig. 1. The field covered in the region of King 5 by the optical photometry of Phelps et al. (1994). The inner region shows the mosaic of the four fields observed by us in the IR. North is up, East on the left

\section{Observations and data reduction}

$J(1.2 \mu \mathrm{m})$ and $K(2.2 \mu \mathrm{m})$ photometry of King 5 was obtained at the $1.5 \mathrm{~m}$ Gornergrat Infrared Telescope (TIRGO) equipped with Arcetri Near Infrared Camera (ARNICA) in October 1997. ARNICA is based on a NICMOS3 $256 \times 256$ pixels array (gain $=20 \mathrm{e}^{-} / \mathrm{ADU}$, read-out noise $=50 \mathrm{e}^{-}$, angular scale $=1^{\prime \prime} /$ pixel, and $4 \times 4^{\prime 2}$ field of view). More details about the observational equipment and infrared camera, and the reduction procedure can be found in Carraro et al. (1999a). Through each filter 4 partially overlapping images of each field were obtained, covering a total field of view of about $8 \times 8^{\prime 2}$, in short exposures to avoid sky saturation. One field is located close to the center of King 5. In addition, a sample of the background population of King 5 was obtained observing a field of $2^{\prime} \times 2^{\prime}$ at about $15^{\prime}$ South-East from the cluster center and a second one of the same dimensions located at about $5^{\prime}$ Northward.

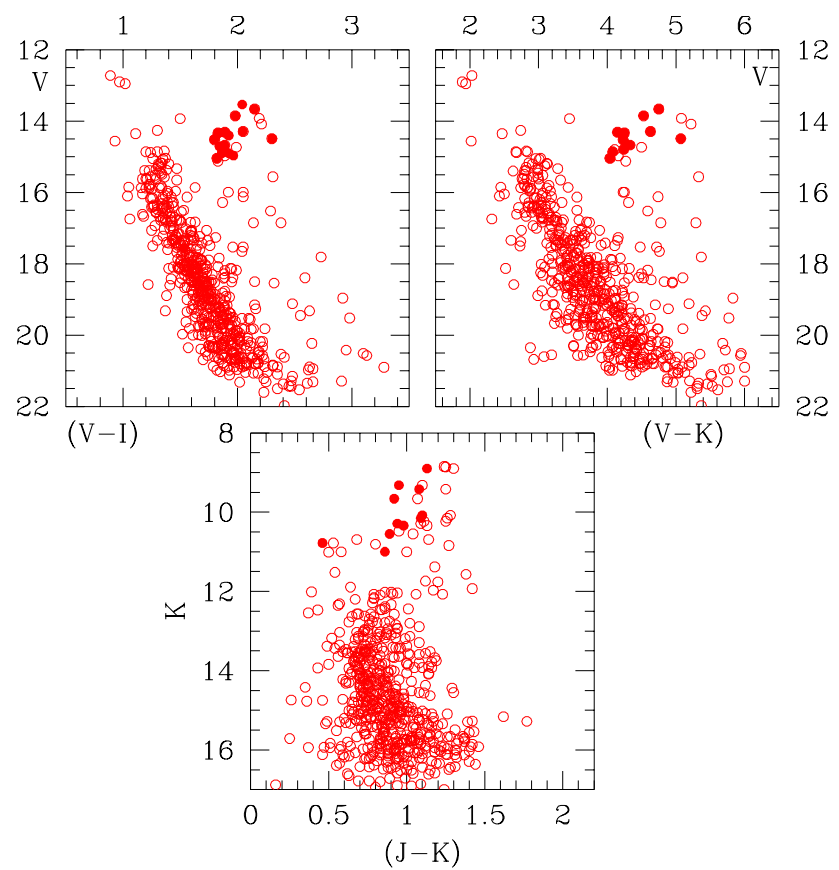

Fig. 2. The CMD of King 5. The optical photometry $(V-(V-$ $I$ ), upper left panel) is taken from Phelps et al. (1994). The IR photometry (lower panel) is that presented in this paper, whereas the upper right panel presents the derived CMD in $V-(V-K)$. Filled circles represent cluster members on the basis of the radial velocity

The log-book of the observations is presented in Table 1 where the centers of the observed fields and the total exposure times are given. The nights were photometric with a seeing of $1^{\prime \prime}-1.5^{\prime \prime}$. Figure 1 presents the final mosaic of the 4 frames for King 5 in $K$ passband.

The conversion of the instrumental magnitude $j$ and $k$ to the standard $J, K$ was made using stellar fields of standard stars taken from Hunt et al. (1998) list. About 10 standard stars per night have been used. In addition 2 stars (thereafter K5A and K5B) inside the field have been measured with a photometer and used for the calibration. Results are summarized in Table 2.

The relations in usage per $1 \mathrm{~s}$ exposure time are:

$J=j+19.51$

$K=k+18.94$

with standard deviation of the zero points of 0.03 mag for the $J$ and 0.04 for the $K$ magnitude. This error is only 


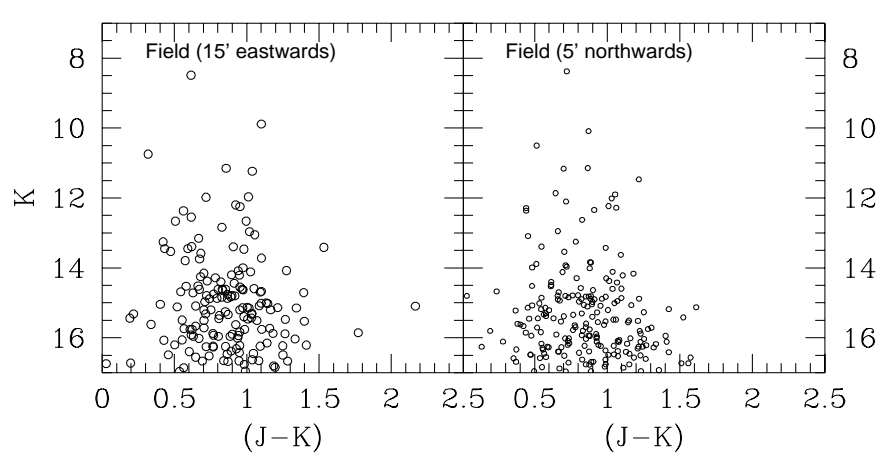

Fig. 3. The CMD of two comparison fields taken $15^{\prime}$ eastwards (left panel) and $5^{\prime}$ northwards (right panel) of King 5

Table 2. Photometry of K5A and K5B

\begin{tabular}{ccc}
\hline \hline Star & $K$ & $J$ \\
\hline K5A & $9.20 \pm 0.04$ & $10.41 \pm 0.05$ \\
K5B & $8.74 \pm 0.04$ & $10.10 \pm 0.05$ \\
\hline \hline
\end{tabular}

due to the linear interpolation of the standard stars. The calibration uncertainty is dominated by the error due to the correction from aperture photometry to PSF fitting magnitude. The standard stars used for the calibration do not cover the entire colour range of the data, because of the lack of stars redder than $(J-K) \sim 0.8$. From our data, no colour term is found for $K$ mag, whereas we cannot exclude it for the $J$ magnitude. Taking all into account, we estimate that the total error on the calibration is about 0.1 mag both in $J$ and $K$ pass-bands.

\section{The color magnitude diagrams}

The CMD derived from optical photometry is shown in the upper left panel of Fig. 2. The global morphology resembles the CMD of an intermediate age open cluster like NGC 2477 (Hartwick et al. 1972), Tombaugh 1 (Carraro \& Patat 1995) and NGC 6603 (Bica et al. 1993). The MS extends vertically down to $V \approx 22$, and the Turn Off point (TO) is situated at $V=15.5,(V-I)=1.3$. The brightest stars in the red region of the diagram can represent the Red Giant (RG) clump of core He-burning stars. Most of these are cluster members, on the basis of their radial velocities (Scott et al. 1995). The Hertzsprung gap is clearly defined. The population of stars on the right side of the MS are probably interlopers, showing that the CMD is contaminated by stars from the field of the Galactic Disk. Also the stars above the TO probably belong to the field.

The CMD from IR photometry is shown in the lower panel of Fig. 2, and appears significantly different. In fact it is quite similar to the CMD of old open clusters like M 67 (Montgomery et al. 1993) and Berkeley 39 (Kassis et al. 1997). The MS extends down to $K=16.5$, and the

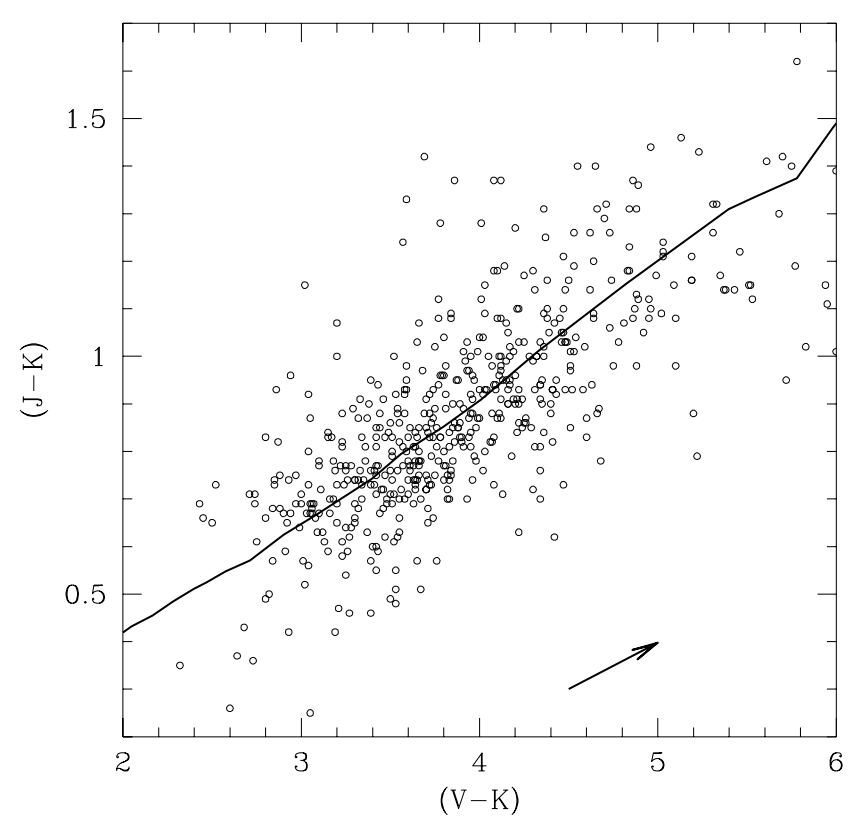

Fig. 4. Two colors diagram for MS stars in King 5. The solid line is a Zero Age MS for $[\mathrm{Fe} / \mathrm{H}]=-0.38$. In the lower left corner the reddening vector is shown. See text for any detail

TO is located at $K=12.5,(J-K)=0.70$. The region above the TO might contain some interlopers (see Fig. 3).

Apparently there is no Hertzsprung gap, and the RGB is sparsely populated, like in all clusters with age greater that 4 Gyr. The stars at $K \approx 10.3,(J-K) \approx 1.1$ might represent the clump of core He burners, quite small in this age range. We recall that IR photometry covers all the cluster, and the foreground contamination is negligible, as it can be seen in Fig. 3, where two comparison fields $15^{\prime}$ eastwards and $5^{\prime}$ northwards, respectively, are shown.

In order to clarify the nature and age range of this cluster, and whether the differences are simply due to the use of different colors, we have combined optical and IR data to construct the CMD in $V-(V-K)$, which is shown in the lower right panel Fig. 2. This CMD looks quite similar to the optical CMD shown in the same figure, demonstrating that King 5 is an intermediate age open cluster. To better assess this point, in the next sections we are going to determine in details the cluster basic parameters.

\section{Reddening}

In order to derive the interstellar extinction for King 5, we have combined optical and IR photometry (600 stars in total), and construct two color diagrams, namely $(J-K)$ vs. $(V-K)$ and $(V-I)$ vs. $(V-K)$, which are shown in Fig. 4 and Fig. 5, respectively. Only MS stars are considered. We superimposed a Zero Age MS (ZAMS) for the 


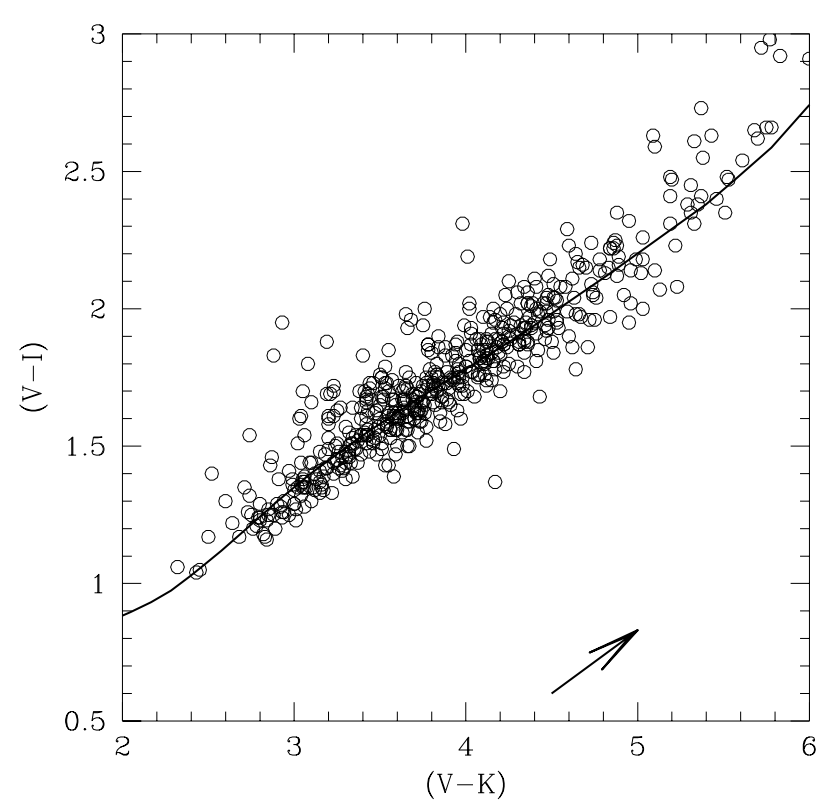

Fig. 5. Two colors diagram for MS stars in King 5. The solid line is a Zero Age MS for $[\mathrm{Fe} / \mathrm{H}]=-0.38$. In the lower left corner the reddening vector is shown. See text for any detail

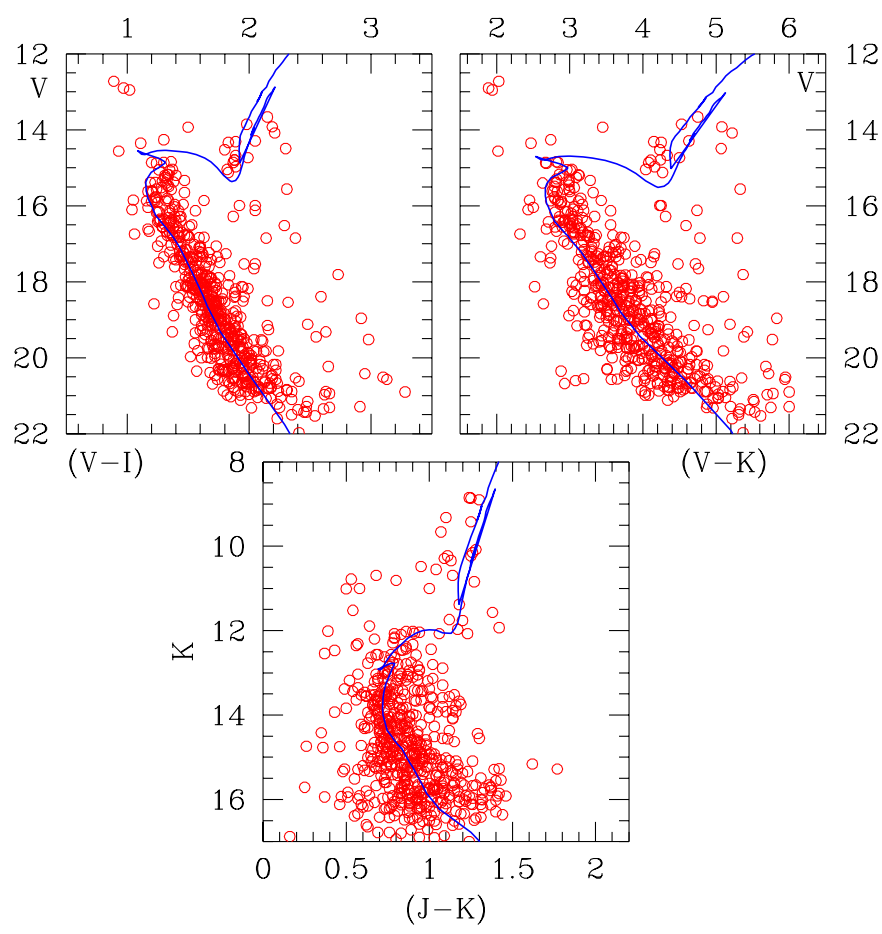

Fig. 6. Age determination for King 5. Overimposed on the CMDs is a $Z=0.008$ isochrone for an age of 1 Gyr. Lower panel shows the fit in the plane $K-(J-K)$, upper left panel in the plane $V-(V-I)$, and upper right panel in the plane $V-(V-K)$. See the text for any detail theoretical metal content $Z=0.008$, obtained translating the observed $[\mathrm{Fe} / \mathrm{H}]$ by means of the relation:

$$
[\mathrm{Fe} / \mathrm{H}]=\log \frac{Z}{0.019}
$$

taken from Bertelli et al. (1994). Since the cluster does not exhibit a RGB, it is not possible to derive an independent photometric estimate for the metal content.

In Fig. 4 the fit has been obtained shifting the ZAMS with $E(J-K)=0.50$ and $E(V-K)=2.45$, which corresponds to a ratio $\frac{E(V-K)}{E(J-K)}=4.9$, relatively close to the value 5.3 suggested by Cardelli et al. (1989).

The fit in Fig. 5, on the other hand, has been achieved shifting the ZAMS by $E(V-I)=1.10$ and $E(V-K)=2.45$, whose ratio turns out to be $\frac{E(V-I)}{E(V-K)}=0.44$, in agreement with the value 0.42 from Cardelli et al. (1989). Although reasonable, these estimates are affected by the limitation that the reddening vector is almost parallel to the ZAMS. However, at least for $E(V-I)$, our results do not differ to much from Durgapal et al. (1998), although these authors did not use the spectroscopic metallicity from Friel et al. (1995). Indeed, by using a lower metallicity, one should expect a larger reddening.

\section{Age and distance}

The knowledge of metallicity and reddening, allows us to infer the distance and age of King 5 by means of fitting with isochrones (Bertelli et al. 1994). The fit is shown in the three panels of Fig. 6, for the planes $V$ vs. $(V-I), V$ vs. $(V-K)$, and $K$ vs. $(J-K)$, respectively.

We have adopted the theoretical metallicity $Z=0.008$ above derived, and performed the fitting with a $1.0 \mathrm{Gyr}$ isochrone, which better matches the observational data. The criterion that guided us in performing this comparison was the simultaneous fit of the TO and the clump magnitudes. Since no membership exists for this cluster, it is not possible to define exactly the MS TO, which is populated also by unresolved binary stars, and interlopers (see the field population in Fig. 3 for an interpretation of the TO region in the IR CMD). However the clump luminosity can be reliably determined, since many stars are also spectroscopic members.

By using the reddening estimated derived in the previous section, the apparent distance moduli $(m-M)_{K,(J-K)}$ and $(m-M)_{V,(V-I)}$, in the plots turn out to be 11.90 and 14.00, respectively. The latter values can also be obtained assuming that the mean clump magnitude is $M_{V}=0.80$ (Girardi et al. 1998). Once corrected, these values converge to the absolute distance modulus $(m-M)_{0}=11.40 \pm 0.15$.

King 5 turns out to be $1.9 \mathrm{kpc}$ distant from the Sun, and about $9.6 \mathrm{kpc}$ far from the Galactic Center. 


\section{Conclusions}

In this paper we have presented a detailed study of the poorly known intermediate age open cluster King 5. By combining optical and IR photometry we have proved that King 5 is a moderate age open cluster about 1 Gyr old, intermediate in age between the Hyades and NGC 752.

By studying the CMD and two color digrams, we have obtained estimates for the cluster reddening and distance. In detail, we found that the color excesses $E_{(J-K)}, E_{(V-I)}$ and $E_{(V-K)}$ are $0.50,1.10$ and 2.45 , respectively, and that their ratios are in agreement with the standard values (Cardelli et al. 1989). The derived corrected distance modulus $(m-M)_{0}=11.40$ implies a distance of $1.9 \mathrm{kpc}$ from the Sun.

Acknowledgements. G.C. acknowledges useful e-discussions with Prof. Ken Janes, and the anonymous referee who helped to improve the presentation of the paper. This study has been financed by the Italian Ministry of University, Scientific Research and Technology (MURST) and the Italian Space Agency (ASI).

\section{References}

Bertelli G., Bressan A., Chiosi C., Fagotto F., Nasi E., 1994, A\&AS 106, 275

Bica E., Ortolani S., Barbuy B., 1993, A\&A 270, 117
Cardelli J.A., Clayton J.C., Mathis J.S., 1989, ApJ 345, 245

Carraro G., Patat F., 1995, MNRAS 276, 563

Carraro G., Vallenari A., Girardi L., Richichi A., 1999a, A\&A 343,825

Carraro G., Girardi L., Chiosi C., 1999b, MNRAS 309, 430

Durgapal A.K., Pandey A.K., Mohan V., 1998, Bull. Astron. Soc. India 26, 551

Friel E.D., Janes K.A., Hong L., Lotz J., Tavarez M., 1995, in "The Formation of the Milky Way", Alfaro E.J. \& Delgato A.J. (eds.). Cambridge University Press, p. 189

Girardi L., Groenewegen M.A.T., Weiss A., Salaris M., 1998, MNRAS 301, 149

Hartwick F.D.A., Hesser J.E., McClure R.D., 1972, ApJ 174, 557

Hron J., 1987, A\&A 176, 34

Hunt L.K., Mannucci F., Testi L., et al., 1998, AJ 115, 2594

Hunt, L., Testi, L., Borelli S., Maiolino, R., Moriondo G., 1994, Arcetry Observatory Technical Report No. 4/94

Kassis M., Janes K.A., Friel E.D., Phelps R.L., 1997, AJ 113, 1723

Montgomery K.A., Marschall L.A., Janes K.A., 1993, AJ 106, 181

Phelps R.L., Janes K.A., Montgomery K.A., 1994, AJ 107, 1079

Scott J.E., Friel E.D., Janes K.A., 1995, AJ 109, 1706

Vallenari A., Richichi A., Carraro G., Girardi L., 1999a, A\&A 349,825

Vallenari A., Carraro G., Richichi A., 1999b, A\&A (in press) 